\title{
The multiple-weighting-systems hypothesis: Theory and empirical support
}

\author{
Dragan Rangelov • Hermann J. Müller • \\ Michael Zehetleitner
}

Published online: 9 December 2011

(C) Psychonomic Society, Inc. 2011

\begin{abstract}
Observers respond faster when the task-relevant perceptual dimension repeats across consecutive trials (e.g., color-color) relative to when it changes (orientationcolor) - the phenomenon termed the dimension repetition effect (DRE). Similarly, when two (or more) different tasks are made to vary randomly across trials, observers are faster when the task repeats, relative to task changesthe phenomenon termed task-switch cost (TSC). Hitherto, the DRE and TSC effects have been discussed independently of each other. Critically, either effect was explained by assuming a single mechanism giving rise to DREs or TSCs. Here, we elaborate strong conceptual similarities between the DRE and TSC effects; we introduce the concept of criterion-specific intertrial sequence effects, with DREs and TSCs being different manifestations of criterionspecific effects. Second, we review available evidence suggesting that none of the single mechanism explanations can readily account for all the findings in the literature. Third, we elaborate on the multiple-weighting-systems (or MWS) hypothesis, a recently proposed account that postulates the existence of several, independent mechanisms sensitive to intertrial sequences. Finally, we test predictions derived from the MWS hypothesis in two novel experiments
\end{abstract}

D. Rangelov $\cdot$ H. J. Müller $\cdot$ M. Zehetleitner Ludwig-Maximilians-Universität München, München, Germany

D. Rangelov $(\triangle)$

LMU Department Psychologie,

Allgemeine \& Experimentelle Psychologie, Leopoldstr. 13,

DE-80802 München, Germany

e-mail: rangelov@psy.lmu.de

H. J. Müller

Birkbeck College (University of London),

London, United Kingdom and discuss the results from both the single- and multiplemechanism perspectives.

Keywords Dimension repetition effect · Task switching . Multiple weighting systems · Dimensional weighting

\section{Introduction}

What we did recently has a substantial effect on our current performance. For instance, we are likely to set a table faster when we put all plates down first, as compared with setting down plates and cutlery in mixed order. Conceptually, one can think of three broadly defined processes that are potentially facilitated by such action sequencing: (1) visual selection (e.g., detecting plates among cups and bowls), (2) perceptual or semantic analysis (e.g., deciding whether the selected plate belongs to the appropriate set), and (3) action execution (e.g., transferring the plate to the table). When setting the table, all three processes (selection, analysis, action) are involved, thus making it difficult to isolate the specific cognitive processes affected by recent experience. Evaluating the role of different cognitive processes as a source of history effects on human performance is the main focus of the present study.

Typically, history effects are investigated by examining changes in a dependent variable as a function of the intertrial sequence - that is, repetitions versus changes of stimulus and/or response properties across consecutive trials. Although many such effects have been described in the literature, they can be broadly classified as being either (1) feature specific or (2) criterion specific in type. Feature-specific intertrial effects relate to repetition/changes of specific paradigm properties: changes in the exact defining feature of the stimulus (e.g., from green to red) or changes in the required response (e.g., from a left- to a right-buttonpress). By 
contrast, criterion-specific effects relate to more abstract changes in task-relevant stimulus properties (e.g., switching from color to orientation as critical stimulus property) or changes in stimulus-response (S-R) mappings (e.g., rom manual to vocal responding).

The present article focuses primarily on criterion-specific intertrial effects. To set the stage, first, different types of criterion effects are outlined and their conceptual similarity is elaborated. Next, unresolved discrepancies between dominant accounts of criterion-specific intertrial effects, all postulating a single mechanism underlying these effects, are elaborated, and several recently proposed, integrative accounts are outlined - with a focus on our own account, which postulates the existence of multiple mechanisms capable of producing criterion-specific intertrial effects. Finally, the results of two new experiments designed to test opposing predictions derived from single- and multiplemechanism accounts, respectively, are presented, which provide a further empirical validation of the MWS hypothesis.

\section{Criterion-specific intertrial effects}

Criterion-specific effects have historically been investigated in two different contexts: as dimension-specific intertrial effects in visual search paradigms and as task-switching effects in paradigms designed to investigate executive functions. Dimension repetition effects ${ }^{1}$ (henceforth, abbreviated as DREs) in visual search tasks are frequently observed in paradigms requiring search for a singleton (odd-one-out) item in multi-item displays. On some trials, the display contains one item that is different from the others in some respect (e.g., a blue bar among green bars). The task may be to discern the presence versus the absence of the singleton (detection task), determine its location (localization task), or discriminate another property such as its orientation (discrimination task). Importantly, the dimension of distinction between the singleton and the other items can be made to vary randomly across trials - for example, from a blue singleton (i.e., a color target) on one trial to a tilted singleton (i.e., an orientation target) on the next. That is, the criterion for selecting the task-relevant item varies across consecutive trials: the singleton dimension can either repeat or change. Previous research has repeatedly demonstrated shorter reaction times (RTs) for dimension repetitions relative to dimension changes (Fecteau, 2007; Found \&

\footnotetext{
${ }^{1}$ An informed reader might notice that in the previous publications, we used the term dimension repetition benefit (and, accordingly, the DRB acronym). Since our paradigm has no baseline condition (when the task relevant dimension neither changes nor repeats across trials) against which benefits or costs incurred by dimension repetitions could be assessed, we decided to henceforth use a more descriptive term: dimension repetition effect and, accordingly, the DRE acronym.
}

Müller, 1996; Müller, Heller, \& Ziegler, 1995; Müller \& Krummenacher, 2006a, 2006b; Olivers \& Humphreys, 2003; Töllner, Gramann, Muller, Kiss, \& Eimer, 2008). Importantly, RTs are almost as short when the exact singleton feature changes but the dimension repeats (e.g., green $\rightarrow$ blue) as for precise singleton feature repetitions (e.g., blue $\rightarrow$ blue). The finding that DREs persist across feature changes argues in favor of their criterion, rather than feature, specificity.

Several mechanisms have been proposed to explain the DREs, which can be broadly divided into accounts based on (1) visual selection (2) response selection, respectively. According to the former, DREs reflect changes in processes leading to the selection of the task-relevant item from a set of multiple (distractor) items. For example, the dimensionweighting account (or DWA; e.g., Müller \& Krummenacher, 2006b) assumes that the buildup of saliency signals on the search-guiding master map of saliencies is facilitated when the previously relevant singleton dimension repeats, relative to when it changes, giving rise to DREs. According to the latter, response selection accounts, DREs reflect changes in postselective processes, such as choosing a task-appropriate response to the already selected stimulus (e.g., Cohen \& Magen, 1999; Cohen \& Shoup, 1997, 2000).

Over the years, proponents of both pre- and postselective accounts have produced evidence that either visual or response selection processes could be influenced by the sequence of task-relevant dimensions across trials. Support for selection-based accounts comes from the fact that (1) DREs are typically observed for multi-item displays - that is, when the task requires search for the task-relevant item prior to selecting a response; (2) DREs are observed for response-irrelevant (but selection-relevant) stimulus properties in compound-search tasks, where the target is selected based on one property, such as its color, but the response is based on another property, such as its orientation (e.g., Becker, 2008; Fecteau, 2007; Olivers \& Humphreys, 2003); and (3) dimension repetition influences very early ERP components related to the sensory processing of the stimulus displays (i.e., the N1; Gramann, Töllner, \& Müller, 2010), as well as markers of spatial-attentional selection (i.e., the N2pc component; Töllner et al., 2008).

The strongest evidence that response selection processes can be affected by dimensional sequence comes from paradigms in which the visual selection component is minimized, such as when a single display item is presented at a fixed location throughout the experiment (Mortier, Theeuwes, \& Starreveld, 2005). Mortier and colleagues devised a variant of the singleton detection task in which an item of a particular shape (triangle), color (red), or size (large) was considered to be a shape, color, or size target, respectively (requiring a target-present response), whereas the distractor was always a small gray circle (requiring a target-absent response). With regard to response mapping, 
the task was comparable to the singleton detection task (i.e., singleton present/absent), with the difference that, by virtue of displays containing a single stimulus at a fixed location, no search for the task-relevant item was necessary. Across two target-present trials, the dimension of distinction between a target and the distractor could either repeat or change, thus potentially giving rise to DREs. And indeed, Mortier et al. did find robust DREs in this non-search detection task-which, they convincingly argued, could not originate from the stimulus selection stage, thus leaving only postselective processing stages as the source of the DREs. On the basis of qualitative similarities between the DREs observed in the search and non-search variants of the detection task, Mortier et al. interpreted the DREs observed in search tasks as being generated, too, at (postselective) stages involved in response selection. Thus, on the basis of the evidence reviewed above, the debate as to the origin of DREs remained unresolved, since neither the visual-selection- nor the responsebased approach could provide a coherent account for the full body of findings reported thus far.

\section{Integrative accounts on DREs}

More recently, there have been several attempts at integrating the seemingly disparate findings regarding the origin of DREs - in particular, (1) the ambiguity resolution account (Meeter \& Olivers, 2006; Olivers \& Meeter, 2006, 2008), (2) the perceptual identification account (Becker, 2010; Krummenacher, Grubert, \& Müller, 2010), and (3) the multiple-weighting systems hypothesis (Rangelov, Müller, \& Zehetleitner, 2011a, 2011b).

The ambiguity resolution account was primarily developed to "provide a comprehensive framework for when intertrial priming does and when it does not occur" (Olivers \& Meeter, 2006, p. 26). According to this account, intertrial priming effects become functional (i.e., beneficial to performance) and measurable only in paradigms in which there is ambiguity (uncertainty) with regard to certain aspects of the task. Ambiguity can relate to (1) selection ambiguity, where it is decided that the selected item (among other items) really is the task-relevant stimulus; (2) response ambiguity, where it is decided on what the task-appropriate response to the selected item should be; and (3) task ambiguity, where it is decided whether the set of currently active responses is appropriate for the task. Olivers and Meeter (2006) argued that selection ambiguity in search detection tasks (discerning the presence/absence of a singleton target) is greater by virtue of singletons being absent on (typically) half the trials; by contrast, selection ambiguity is smaller in compound-search tasks, since a singleton is present on every trial. Thus, differences in selection ambiguity could account for the differences in
DRE magnitude typically observed between search detection (strong DREs) and compound-search tasks (weaker DREs). Consistent with this, increasing selection ambiguity in compound-search tasks by introducing singleton-absent no-go trials was found to increase the DREs, as compared with the standard compound task. In summary, according to the ambiguity account, the amount of ambiguity in a paradigm determines the magnitude of DREs. The ambiguity itself can originate at different processing stages, thus accounting for DREs observed in both search detection (from selection ambiguity) and non-search detection tasks (from response ambiguity).

Another account proposed by Becker (2010; see Krummenacher et al., 2010, who proposed a similar mechanism) to explain the disparate findings relating to DREs in different, search and non-search, paradigms assumes that DREs originate from postselective processes prior to response selection stages - that is, perceptual identification of the selected (target) item. In support of this account, Becker reports an experiment in which participants were required, in separate sessions, to either make a saccade to a target singleton (if present) or report the singleton's presence by a manual buttonpress. While substantial DREs were observed for manual RTs, only slow, but not fast, latency saccades were affected by dimensional sequence. Becker assumed that fast saccades are driven directly by the target's saliency, with the computation of saliency signals being insensitive to dimensional sequence, whereas slow saccades and manual detection responses reflect, besides target saliency, (slower) processes of target identification, which are sensitive to dimensional sequence. That is, Becker (2010) ascribed the origin of DREs to postselective processes of target identification. This perceptual identification hypothesis could account for DREs observed in both search and non-search paradigms, given that dimension-sensitive identification processes are involved in both tasks.

\section{The multiple-weighting-systems hypothesis}

The MWS assumes the existence of several independent mechanisms, situated at different processing stages, which can all give rise to criterion-specific effects. Which weighting mechanism is active depends on specific paradigm properties. A paradigm is specified by (1) the stimuli it uses, (2) the response criterion - that is, the stimulus property (or properties) that must be encoded for selecting a response, and (3) the actual responses that are required. These paradigm specification criteria are illustrated in Fig. 1.

The paradigm properties directly influence which cognitive processes are necessary for performing a particular task. As was mentioned above, these processes can be grouped 
Paradigm properties

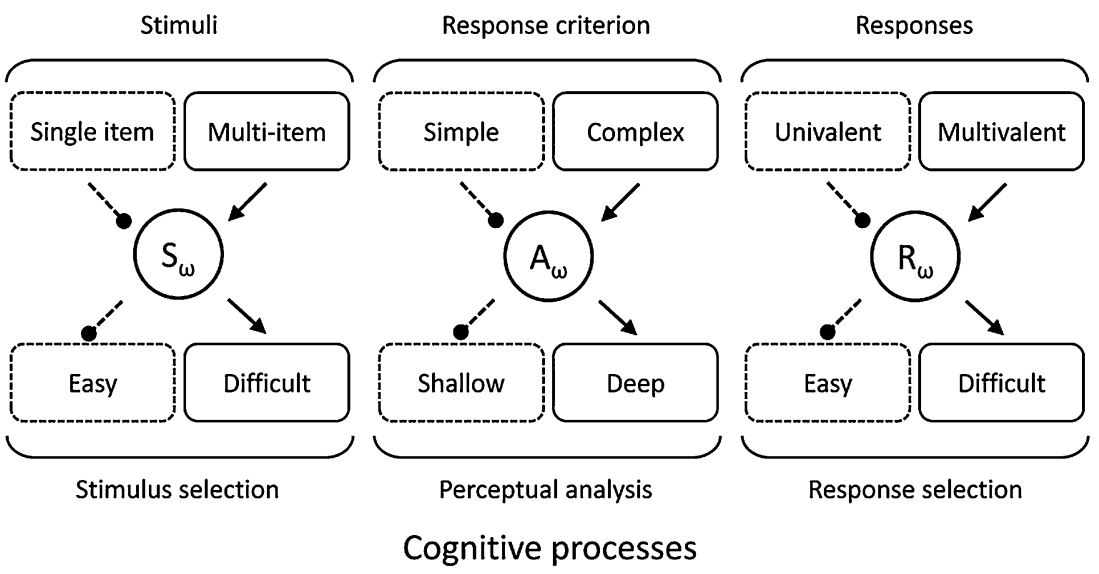

Fig. 1 Mapping between paradigm properties and cognitive processes. A paradigm is defined by its stimuli, by the response criterion (i.e., what stimulus property must be encoded for selecting a response), and by the required responses. There are three broad groups of cognitive processes: visual selection, perceptual analysis, and response selection. The stimulus material (single- vs. multiple-item displays) influences the visual selection processes (easy vs. difficult); the response criterion (simple vs. complex) influences perceptual analysis processes

into three broad categories, relating to (1) stimulus selection, (2) perceptual or semantic analysis of the selected stimuli, and (3) response selection processes. On the basis of the stimulus material, stimulus selection can be either easy, as in single-item displays, or relatively difficult, as in multi-item displays. A particular response criterion either can require shallow perceptual analysis, as in the singleton detection task in which merely discerning the presence of a unique, odd-one-out stimulus suffices to produce a response, or can require deep perceptual analysis, as when features of the selected stimulus have to be determined prior to responding. Finally, the response selection processes can be either easy, when the responses are univalent (i.e., one stimulus is mapped to one response), or relatively difficult, when the responses are multivalent (i.e., several stimuli are mapped to one response).

The core assumption of the MWS hypothesis is that different cognitive processes are associated with different weighting mechanisms, represented in Fig. 1 as $\mathrm{S}_{\omega}, \mathrm{A}_{\omega}$, and $\mathrm{R}_{\omega}$ for the mechanisms associated with stimulus selection, perceptual/semantic analysis, and response selection, respectively. The three mechanisms are all sensitive to intertrial sequences, thus potentially giving rise to intertrial effects. Importantly, as is suggested by the evidence reviewed below, these three mechanisms are independent of each other, and state changes in one of them do not affect the state of other systems.

The (stimulus) selection weighting system $\left(\mathrm{S}_{\omega}\right)$ modulates the efficiency with which feature contrast signals in the various perceptual dimensions influence overall-saliency (shallow vs. deep); and the required responses (univalent vs. multivalent) influence response selection processes (easy vs. difficult). Associated with the different processes (selection, analysis, responding) are different sequence-sensitive systems: $\mathrm{S}_{\omega}, \mathrm{A}_{\omega}$, and $\mathrm{R}_{\omega}$, respectively. Different paradigm properties can influence the state of these systems (full connectors) or not (dashed connectors), with these changes subsequently influencing related cognitive processes. See the text for details

coding. With single-item displays, feature contrast signals are generated in multiple dimensions (i.e., there are no signals generated uniquely in one dimension): a single yellow, vertical bar on a black background would differ from its surroundings in luminance, color, and orientation. Consequently, the $\mathrm{S}_{\omega}$ is not affected by dimension sequence in single-item displays (dashed connectors to $\mathrm{S}_{\omega}$ in Fig. 1). By contrast, paradigms using multi-item displays may involve modulations of the state of the $\mathrm{S}_{\omega}$ (full line to $S_{\omega}$ in Fig. 1). The $S_{\omega}$, in turn, influences performance in paradigms in which stimulus selection is difficult; the mechanism underlying this influence has been elaborated within the DWA (Müller \& Krummenacher, 2006a, 2006b).

The second weighting system $\left(\mathrm{A}_{\omega}\right)$ influences processes of perceptual/semantic analysis of the selected items. The $\mathrm{A}_{\omega}$ system is engaged in tasks that involve complex response criteria and, thus, require deep analysis of the selected stimulus prior to deciding upon a response. By contrast, tasks with simple response criteria and shallow analysis demands would be little influenced by the $\mathrm{A}_{\omega}$ system.

With regard to the exact mechanism via which $\mathrm{A}_{\omega}$ modulates the dynamics of perceptual analysis, several alternatives are possible. One, argued for by Krummenacher et al. (2010; see also Becker, 2010), would be that the processes of feature identification are speeded for the previously relevant stimulus dimension, relative to the previously irrelevant dimension: identification of a repeated feature is expedited because analysis starts with the same, specific feature in the dimension that led to a successful 
response on the last trial. Alternatively, the $\mathrm{A}_{\omega}$ system may be based on task set representations, with DREs observed in paradigms with complex response criteria reflecting task set reconfiguration processes (e.g., Rogers \& Monsell, 1995). For example, the non-search detection task of Mortier et al. (2005) requires discrimination between a distractor and targets that can differ from the distractor in one of several possible ways (e.g., in color, shape, or size). Thus, detecting a color target could be thought of as a color discrimination task, whereas detecting a shape target would constitute a shape discrimination task. Consequently, changing the dimension of discrimination across trials would introduce a task change as well, so that task change/repetition sequences are perfectly correlated with dimension change/repetition sequences. An important difference between the task switch cost and identification facilitation accounts is that according to the former, DREs would arise owing to active task set reconfiguration processes, whereas according to the latter, DREs occur due to the biasing of the order of identifying different stimulus attributes. In summary, the DREs in paradigms with complex response criteria could reflect either facilitated feature identification or task set reconfiguration processes (or both). The MWS hypothesis, at present, is agnostic as to the exact mechanism; it essentially states that the processes underlying the $\mathrm{A}_{\omega}$ system are separate from those underlying the $\mathrm{S}_{\omega}$ system.

The third hypothesized system is associated with the processes of response selection $\left(\mathrm{R}_{\omega}\right)$. The reason for postulating such a system derives from the work carried out within the framework of response-based accounts of DREs (e.g., Cohen \& Magen, 1999; Mortier et al., 2005). Additionally, findings from the dual-task and task-switching literature show that dual-task costs or TSCs increase if the two tasks to be performed involve the same response sets (i.e., multivalent mapping), relative to tasks using nonoverlapping response sets (Gade \& Koch, 2007; Koch, Gade, Schuch, \& Philipp, 2010; Mayr, 2001). Furthermore, investigation of ERP markers of effector-specific response sequence effects revealed these to be independent of the ERP markers of dimension sequence effects in a paradigm that used a compound-search task with uncorrelated dimension and response sequences (Töllner et al., 2008) Finally, behavioral studies have demonstrated that across task switch trials, response repetition produces inhibitory effects additive to facilitatory effects of stimulus repetition (Druey \& Hübner, 2008).Taken together, these findings argue in favor of a separable response selection weighting $\left(\mathrm{R}_{\omega}\right)$ system.

Testing integrative accounts of DREs

If a particular paradigm involves any of the hypothesized sources of DREs (ambiguity, identification processes, or a weighting system), significant criterion-specific sequence effects are expected. Consequently, DREs observed in single-task paradigms (whether of the search or the nonsearch type) could be accounted for by assuming either single (e.g., identification processes) or multiple (selection, analysis, response-based) weighting mechanisms. However, examining for DREs across trials of different tasks would provide evidence in favor of, or against, the MWS hypothesis. If the tasks in question engage identical weighting systems, significant DRBs are expected across trials of different tasks. By contrast, if the weighting systems differ between tasks, no DRBs are expected to arise. The same hypothesis, by virtue of using a task switch paradigm, could be reformulated as follows: If two tasks share a hypothesized weighting system, the costs of switching between them should be smaller with dimension repetitions, relative to dimension changes. The MWS hypothesis essentially predicts an interaction between task and dimension sequence such that DREs should persist across task switches or that TSCs should be lower with dimension repetitions, provided the tasks in question share a hypothesized sequence-sensitive (weighting) mechanism.

Several testable predictions with regard to DREs across task switches can be derived from the MWS hypothesis. First, no criterion-specific effects are expected across tasks that engage different weighting systems (e.g., $\mathrm{S}_{\omega}$ and $\mathrm{A}_{\omega}$, respectively), because the mechanisms driving DREs across trials of two such tasks would differ. Second, if two tasks engage the same weighting system, significant criterionspecific effects are expected even across task switches. Third, if a task engages multiple weighting systems (e.g., both $\mathrm{S}_{\omega}$ and $\mathrm{A}_{\omega}$ ), (1) there should be significant criterionspecific effects across this and any other task engaging at least one of the same weighting systems (either $S_{\omega}$ or $A_{\omega}$ ), and (2) repetition of such a task should produce stronger intertrial effects than repetition of a task engaging only one weighting system.

In contrast to the MWS hypothesis, Becker's (2010) account assumes that identification processes drive DREs in both search and non-search tasks. This, in turn, would predict significant DREs across task switches between search (e.g., search detection) and non-search (e.g., nonsearch detection) tasks. As for predictions deriving from the ambiguity resolution account, its current state of theoretical development does not permit any specific hypotheses to be formulated about DREs across task switches, and discussion of this account - in the light of the present findings - is deferred to the General Discussion section.

Rangelov et al. (2011a) tested the first prediction deriving from the MWS hypothesis in a series of three experiments. A task-switching paradigm was used with two tasks that, 
according to the task analysis method illustrated in Fig. 1, engaged primarily either the selection (search detection task; $\mathrm{S}_{\omega}$ ) or the analysis weighting (non-search feature discrimination task; $\mathrm{A}_{\omega}$ ) system. Consequently, on the MWS hypothesis, no criterion-specific effects were expected across trials of different tasks. Data analyses revealed substantial intertrial effects across task repetitions for both tasks, suggesting that some sequence-sensitive system influenced behavior in both tasks. Importantly, however, there were no DREs across task switches, arguing that the weighting systems engaged in the two tasks were indeed different. Further analyses showed that this pattern of results could not be explained by task, display type, or response switch costs.

Rangelov et al. (2011b) tested the second prediction deriving from the MWS hypothesis in another set of three experiments. A task-switching paradigm was used with tasks that could share a weighting system: Experiment 1 used tasks that could both be assumed to engage the selection weighting system (search detection and singleton localization tasks), while Experiment 3 used tasks that both engaged the analysis weighting system (nonsearch detection and non-search feature discrimination tasks). Alternatively, the tasks could engage different weighting systems: In Experiment 2, one task engaged the selection weighting system (search detection task), while the other engaged the analysis weighting system (non-search detection task). On the MWS hypothesis, significant intertrial effects were expected across tasks that shared a weighting system (Experiments 1 and 3), while no effects were expected across tasks that engaged different weighting systems (Experiment 2). The results were fully in line with these predictions: Significant DREs were observed across task switches in Experiments 1 and 3, while there were no DREs across task switches in Experiment 2.

In summary, our previous work demonstrated the persistence of criterion-specific intertrial effects across task switches, but only if the tasks in question shared a hypothesized weighting system. These findings provide strong empirical support for the core assumption of the MWS hypothesis that several, independent mechanisms, situated at different processing stages, are sensitive to dimensional sequences and capable of producing DREs. Most importantlany single-mechanism account of criterion-specific effects (whether it is selection, identification, or response based) would predict a uniform pattern of intertrial effects across task switches; that is, DREs would either always occur or never occur across task switches, which is clearly contradicted by the findings of Rangelov et al. (2011a, 2011b). Thus, arguably, the MWS hypothesis offers the most parsimonious account of the available findings.

\section{Issue of the present study}

Our previous work (Rangelov et al., 2011a, 2011b) confirmed two out of three predictions derived from the MWS hypothesis. The present study was designed primarily to test the third prediction: Tasks engaging multiple mechanisms should exhibit a DRE pattern different from that for tasks engaging only one mechanism.

From the MWS perspective, if a task influences several weighting mechanisms (i.e., both $\mathrm{S}_{\omega}$ and $\mathrm{A}_{\omega}$ ), the DREs observed in this task would have multiple origins. Consequently, one would expect the DREs in such a task to be larger than those observed in tasks that primarily engage only one weighting system: $\left(\mathrm{DRE}_{\mathrm{S} \omega}+\mathrm{DRE}_{\mathrm{A} \omega}\right)>\left(\mathrm{DRE}_{\mathrm{S} \omega}\right.$ $\left.\mathrm{XOR} \mathrm{DRE}_{\mathrm{A} \omega}\right)$. Furthermore, if such a task alternates across trials with a task engaging only one system, there should be significant DREs across task changes regardless of the specific weighting system $\left(\mathrm{S}_{\omega}\right.$ or $\left.\mathrm{A}_{\omega}\right)$ influenced by the latter task. This derives from the fact that across trials of any two tasks, there will be at least one shared dimension-sensitive weighting mechanism, which, as was set out earlier, is a precondition for DREs to arise.

To test the third prediction, a search feature discrimination task was used. This task involved multi-item displays containing a feature singleton on every trial (defined in either the color or the orientation dimension), with observers having to report the exact singleton feature (left- vs. right-tilted, or blue vs. green). Conceptually, this search discrimination task entails both difficult selection (involving $\mathrm{S}_{\omega}$ ) and deep perceptual analysis (involving $\mathrm{A}_{\omega}$ ). The search discrimination task was mixed with either a search detection task (difficult selection, shallow analysis; $\mathrm{S}_{\omega}$ ) or a non-search detection task (easy selection, deep analysis; $\mathrm{A}_{\omega}$ ) in Experiments 1 and 2, respectively. Data analysis focused on testing DREs across different tasks and different task sequences (repetition/change).

\section{General method}

The two experiments used similar stimulus material and the same general procedure. For this reason, the methods for both experiments are presented together.

Participants One group of 10 participants (5 female, mean age $=25$ years) and another group, also of 10 participants ( 4 female, mean age $=26$ years), took part in Experiments 1 and 2 , respectively, for either monetary compensation $(8 € / \mathrm{h})$ or course credit. All participants reported normal or corrected-to-normal vision, as well as previous experience with psychophysical studies. They were all naïve with respect to the purpose of the experiments. 
Apparatus The experiments were controlled by a Dell PC running under the Windows XP operating system. The stimuli were presented on a Fujitsu Siemens 21-in. CRT monitor, with a screen resolution of $1,280 \times 1,024$ pixels and a refresh rate of $85 \mathrm{~Hz}$. The experimental software was custom-written in $\mathrm{C}++$. Participants performed the task in a dimly lit and acoustically isolated room, seated in front of the monitor. Head-to-monitor distance was $57 \mathrm{~cm}$, controlled by means of a chinrest. Participants responded by pressing the left or the right button of a computer mouse, with their left or right index finger, respectively.

Stimuli There were two different displays types (illustrated in Fig. 2): (1) single-item displays, with only one bar presented in the screen center, and (2) multi-items displays, with 35 bars arranged in four (virtual) concentric circles made up of $1,6,12$, and 16 bars, respectively. Stimuli were presented on a homogeneous, medium gray background (CIE xyY .283, .311, 31, respectively). Individual bars subtended approximately $0.6^{\circ} \times 2.2^{\circ}$ of visual angle, with multi-item displays subtending approximately $21^{\circ}$ $\times 21^{\circ}$ of visual angle. There were five possible bars: (1) yellow (CIE xyY .378, .518, 83), vertical (considered a distractor bar), (2) yellow, tilted $45^{\circ}$ clockwise from the vertical (right-tilted), (3) yellow, tilted $45^{\circ}$ counterclockwise from the vertical (left-tilted), (4) blue $(.225, .283,83)$, vertical, and (5) green $(.279, .505,83)$, vertical. Left- and righttilted bars (differing from the distractor in orientation) were considered orientation targets, while blue and green bars (differing from the distractor in color) were considered color targets.

Tasks There were three possible tasks: (1) search detection, (2) non-search detection, and (3) search discrimination. In both search- and non-search detection tasks, participants had to discern whether the presented stimulus display contained a target (there was a target on $60 \%$ of the trials) or not (target present/absent) and press a corresponding response button (left/right). Thus, search- and non-search detection tasks were identical in terms of response mappings. The tasks, however, differed in the displays types: The non-search detection task used single-item displays, and the search detection task multi-item displays. The search discrimination

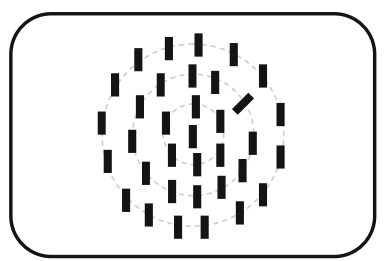

Multiple-item display

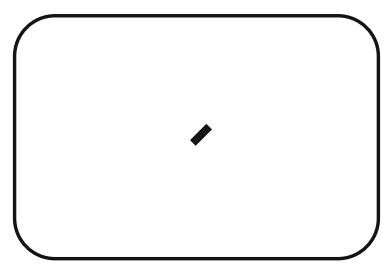

Single-item display
Fig. 2 Illustration of stimulus displays used in the present study task also used multi-item displays, however, with a target present on all trials. Instead of responding target present/ absent, participants had to report the exact feature of the target (left- vs. right-tilted, blue vs. green) by pressing the corresponding response button (left/right). Different features within a dimension of discrimination (e.g., blue vs. green for color) were mapped to different responses, while different features across dimensions (e.g., blue and left-tilted) were mapped to the same response. The task to be performed on a given trial was precued by a task-specific cue word: (1) "detection" for search detection, (2) "identification" for non-search detection, and (3) "discrimination" for the search discrimination task.

Procedure Every trial started with a task cue presented for $1,000 \mathrm{~ms}$. The cue was followed by a stimulus display, which was presented until the participant responded. In case of an incorrect response, the word "error" was presented for 1,000 ms. Between trials, a blank screen was shown for a variable ISI (950-1,050 ms). The trial sequence, along with the respective timings, is illustrated in Fig. 3. There were 35 blocks of 60 trials, resulting in 2,100 trials in total. The first three blocks were considered a practice session and were not included in the analyses. Participants took approximately $2 \mathrm{~h}$ to complete all trials.

Participants were to respond on every trial. Different tasks involved different S-R mappings: (1) target present/ absent for the detection tasks (whether of the search or the non-search variety) and (2) color or orientation discrimination for the discrimination task. There were two possible S-R mappings for detection tasks (present/absent: left/right or present/absent: right/left), and four mappings for the discrimination task: two mappings per dimension (e.g., blue/ green: left/right or blue/green: right/left) $\times$ two dimensions (color and orientation). Two mappings in detection $\times$ four mappings in discrimination tasks resulted in eight possible combinations of S-R assignments, which were counterbalanced across participants.

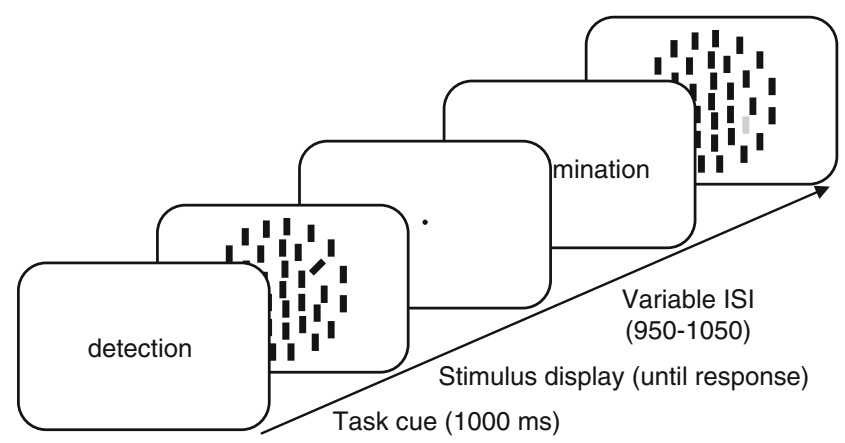

Fig. 3 Illustration of the stimulus sequence and associated presentation times during trials of the present experiments. The real stimuli were colored bars presented on a gray background 
Design and data analyses Every trial was characterized by the task to be performed and by the task-relevant dimension. Tasks and dimensions were randomized across trials. Thus, across consecutive trials, the task and dimension could either repeat or change. This resulted in a 2 (search detection vs. search discrimination task in Experiment 1; non-search detection vs. search discrimination in Experiment 2) $\times 2$ (task repetition vs. change) $\times 2$ (color vs. orientation dimension $) \times 2$ (dimension repetition vs. change) design. The mean RTs were examined by a repeated measures analysis of variance (ANOVA) with main terms for task, task sequence, dimension, and dimension sequence.

\section{Results}

\section{Experiment 1}

Trials of search detection and search discrimination tasks were mixed in Experiment 1. Both tasks used multi-item displays, albeit with different response mappings. Participants made response errors on approximately $6 \%$ of the completed trials. Inspection of the error pattern did not reveal any indications of speed-accuracy trade-offs, and errors were not further analyzed. Trials with a correct response were filtered for extreme RTs (below $200 \mathrm{~ms}$ and above $1,000 \mathrm{~ms}$ ), resulting in the elimination of $2 \%$ of the correct-response trials. The remaining trials were sorted according to task, task sequence, dimension, and dimension sequence into 16 conditions, with, on average, 53 trials (interquartile range, 36-72 trials) per condition per participant.

Inspection of the mean RTs revealed that the detection task was performed overall faster than the discrimination task (473 vs. $538 \mathrm{~ms}$ ); furthermore, RTs were shorter for task repetitions than for changes (488 vs. $523 \mathrm{~ms})$ and were shorter for dimension repetitions compared to changes (DRE: 488 vs. $523 \mathrm{~ms}$ ). These observations were confirmed by a four-way repeated measures ANOVA, which yielded significant main effects of (1) task, $F(1,9)=14.52$, $p<.01, \eta_{\mathrm{p}}^{2}=.61$, (2) task sequence, $F(1,9)=38.58, p<.01$, $\eta_{\mathrm{p}}^{2}=.81$, and (3) dimension sequence, $F(1,9)=23.43$, $p<.05, \eta_{\mathrm{p}}^{2}=.72$.

Analysis of DREs Inspection of DREs revealed this intertrial effect to be stronger for the discrimination than for the detection task (see Fig. 4), which was confirmed by a significant task $\times$ dimension sequence interaction, $F(1,9)=8.38, p<.05, \eta_{\mathrm{p}}^{2}=.48$. Planned $t$-tests revealed the DREs to be significant in both the discrimination task, $t(9)=4.38, p_{\text {one-tailed }}<.05$, and, albeit smaller, the detection task, $t(9)=3.43, p_{\text {one-tailed }}<.05$. The DREs were larger across task repetitions than across task changes (see
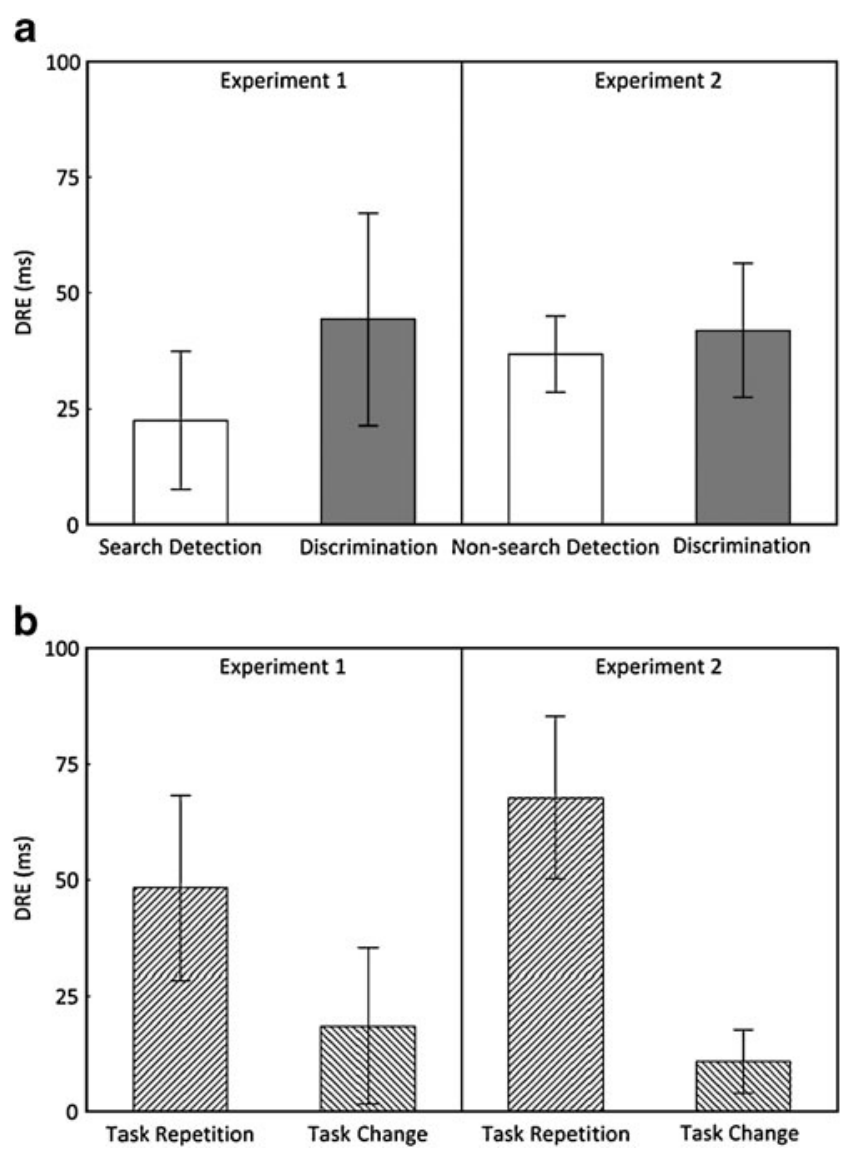

Fig. 4 Mean dimension repetition effects (DREs; $\mathrm{RT}_{\text {different dimension }}-$ $\mathrm{RT}_{\text {same dimension }}$ ) observed in Experiments 1 and 2 a across different tasks (search/non-search detection vs. discrimination), and $\mathbf{b}$ across different task sequences (task repetition vs. change). Vertical lines denote $95 \%$ confidence intervals (CIs); DREs for which zero lies outside the CI are significant at $p<.05$

Fig. 4a), as confirmed by a significant task sequence $\times$ dimension sequence interaction, $F(1,9)=15.94, p<.01$, $\eta_{\mathrm{p}}^{2}=.64$, but were significant in both cases [across task repetitions, $t(9)=5.48, p_{\text {one-tailed }}<.05$; across task changes, $t(9)=2.48, p_{\text {one-tailed }}<.05$; Fig. $\left.4 \mathrm{~b}\right]$.

Other effects The ANOVA also revealed the dimension $\times$ dimension sequence interaction, $F(1,9)=5.29, p<.05$, $\eta_{\mathrm{p}}^{2}=.37$, to be significant. Furthermore, the task $\times$ dimension $\times$ dimension sequence interaction was marginally significant, $F(1,9)=3.68, p=.09, \eta_{\mathrm{p}}^{2}=.29$. Post hoc analyses revealed that these interactions were due to stronger DREs for color $(62 \mathrm{~ms})$ than for orientation (32 $\mathrm{ms}$ ) in the discrimination task. By contrast, DREs were comparable between the two dimensions in the detection task ( 25 and $19 \mathrm{~ms}$, respectively). Finally, the task $\times$ task sequence interaction was significant, $F(1,9)=5.37$, $p<.05, \eta_{\mathrm{p}}^{2}=.37$ : It was more difficult to switch from the discrimination to the detection task (46 ms) than vice versa (24 ms). No other main effects or interactions turned out 
significant (all $F_{\mathrm{S}}<2.32, p \mathrm{~s}>$.16). Importantly, the task $\times$ task sequence $\times$ dimension sequence interaction was nowhere near significance levels, $F(1,9)=0.03, p=.87$, indicating that the task sequence $\times$ dimension sequence interaction was not task specific.

Examination of the task $\times$ dimension sequence interaction separately for the different task sequences revealed a borderline significant interaction for task repetitions, $F(1,9)=5.01, p=.052, \eta_{\mathrm{p}}^{2}=.36$, with DREs of 37 and $65 \mathrm{~ms}$ for search detection and search discrimination, respectively; for task changes, by contrast, the task $\times$ dimension sequence interaction was far from significant, $F(1,9)=1.92, p=.20, \eta_{\mathrm{p}}^{2}=.18$ (DREs of 20 and $34 \mathrm{~ms}$ for search detection and search discrimination, respectively). Taken together, these results indicate that while the DREs for task repetitions are more marked for discrimination relative to detection tasks, the DREs across task switches are independent of the particular sequence in which the two tasks are performed (discrimination $\rightarrow$ detection vs. detection $\rightarrow$ discrimination).

\section{Experiment 2}

Trials of the non-search detection and the search discrimination task were mixed in Experiment 2. As in Experiment 1, the $\mathrm{S}-\mathrm{R}$ mappings differed between tasks; unlike Experiment 1, the stimulus displays also differed across tasks: (1) Single-item displays were used for the non-search detection task, and (2) multi-item displays for the search discrimination task. Participants made an error response on approximately $5 \%$ of all trials. Extreme RTs were produced in approximately $2 \%$ of correct-response trials. The remaining trials were sorted according to task, task sequence, dimension, and dimension sequence, yielding approximately 56 trials (interquartile range, 37-72 trials) per condition per participant.

Inspection of the mean RTs revealed that non-search detection was performed faster than search discrimination (496 vs. $568 \mathrm{~ms}$ ); RTs were shorter for task repetitions than for task changes (510 vs. $554 \mathrm{~ms}$ ) and were shorter for dimension repetitions than for dimension changes (DRE: 512 vs. $552 \mathrm{~ms}$ ). These effects were confirmed by a four-way repeated measures ANOVA, which revealed the main effects of task, $F(1,9)=11.66, p<.01, \eta_{\mathrm{p}}^{2}=.56$, task sequence, $F(1,9)=29.39, p<.01, \eta_{\mathrm{p}}^{2}=.76$, and dimension sequence, $F(1,9)=87.50, p<.01, \eta_{\mathrm{p}}^{2}=.91$, to be significant.

Analysis of DREs DREs were slightly (5 ms) larger for the discrimination task than for the non-search detection task (see Fig. 4a), but this difference was not reliable (nonsignificant task $\times$ dimension sequence interaction:
$F<1, p>.05)$. Furthermore, as is depicted in Fig. 4b, DREs were stronger across task repetitions than across task changes [significant task sequence $\times$ dimension sequence interaction: $\left.F(1,9)=48.96, p<.01, \eta_{\mathrm{p}}^{2}=.84\right]$, but were significant in both cases [task repetitions, $t(9)=8.77, p_{\text {one-tailed }}<.05$; task changes, $\left.t(9)=3.57, p_{\text {one-tailed }}<.05\right]$. No other main effects or interactions proved significant, all $F_{\mathrm{s}}<3.25, \mathrm{ps}>.10$.

Analysis of the task $x$ dimension sequence interaction separately for the different task sequences yielded no reliable interaction effects: task repetitions, $F(1,9)=2.96, p=.12$, $\eta_{\mathrm{p}}^{2}=.25$ (DREs of 58 and $79 \mathrm{~ms}$ for non-search detection and search discrimination, respectively), and task changes, $F(1,9)=.62, p=.45, \eta_{\mathrm{p}}^{2}=.06$ (DREs of 14 and $9 \mathrm{~ms}$, respectively). Thus, the DREs turned out "symmetrical" in Experiment 2, for both task repetitions (detection $\rightarrow$ detection vs. discrimination $\rightarrow$ discrimination) and task switches (discrimination $\rightarrow$ detection vs. detection $\rightarrow$ discrimination).

Between-experiments analyses

Within-experiments analyses revealed the task $\times$ dimension sequence interaction to be significant in Experiment 1, but not in Experiment 2. According to the MWS hypothesis, the DREs should have been larger in the search discrimination task than in the other two tasks (search detection, non-search detection), because in the search discrimination task the DREs would originate from multiple weighting systems, whereas those in the other tasks would have one source only. Thus, a significant task $\times$ dimension sequence interaction was expected in both experiments.

Inspection of the DREs in the search discrimination task showed them to be of comparable magnitude in both experiments (44 and $42 \mathrm{~ms}$ in Experiments 1 and 2, respectively; see Fig. 4a); this was substantiated by an independentsamples $t$-test comparison of the mean DREs between the two experiments $(t<1, p>.05)$. By contrast, for the search detection task (Experiment 1), the DREs were $15 \mathrm{~ms}$ smaller than those for the non-search detection task (Experiment 2, see Fig. 4a); this difference was significant, $t(14)=-1.91$, $p_{\text {one-tailed }}<.05$.

\section{Discussion}

In the present study, the search discrimination task (difficult search, deep analysis; $S_{\omega}$ and $A_{\omega}$ ) alternated randomly across trials, intermixed with either the search detection task (difficult search, shallow analysis; $\mathrm{S}_{\omega}$ ) or the non-search detection task (easy search, deep analysis; $A_{\omega}$ ) in Experiments 1 and 2, respectively. According to the MWS hypothesis, the DREs in the search discrimination task should have been larger (by virtue of having two weighting systems as 
sources) than those in either the search or the non-search detection task (one source). Furthermore, the MWS hypothesis predicted significant DREs across task changes in both experiments, because the tasks used in either experiment always shared at least one weighting system. ${ }^{2}$

In accordance with the MWS hypothesis, the results of both Experiments 1 and 2 showed significant DREs across both task repetitions and task changes. Also in accordance with the MWS hypothesis, the DREs were larger for the search discrimination task than for the search detection task (Experiment 1). Finally, despite a numerical difference of $5 \mathrm{~ms}$ in the predicted direction, there was no significant difference in DRE magnitude between the search discrimination and non-search detection tasks (Experiment 2). Between-experiments analyses showed that the DREs for the discrimination task were comparable across experiments, while the DREs for the search detection task were weaker than those for the non-search detection task.

The MWS hypothesis can account for the present findings in a straightforward manner. There were significant DREs across task repetitions for all tasks because at least one dimension-sensitive mechanism was engaged in every task. There were significant DREs across task switches for both Experiments 1 and 2 because, across any two tasks, there was always at least one shared weighting system. In fact, the present results closely follow the predictions derived from the MWS hypothesis, as illustrated in Figs. 1 and 2.

One prediction deriving from the MWS hypothesis was, however, not confirmed by the data; namely, DREs were expected to be larger in the search discrimination than in the non-search discrimination task. While there was a numerical tendency in the expected direction $(5 \mathrm{~ms})$, this difference was not significant. On the other hand, the DREs for the search discrimination task were comparable across experiments and, in line with MWS predictions, were stronger than the DREs in the search detection task (Experiment 1). This pattern indicates that the large DREs in the non-search detection task (relative to the smaller ones in the search detection task) were responsible for the nonsignificant task $\times$ dimension sequence interaction in Experiment 2 .

While the large DREs in the non-search detection task were not explicitly predicted, they are not entirely surprising. Mortier et al. (2005, Experiment 1) also reported larger DREs in their non-search detection task than

\footnotetext{
2 The MWS hypothesis, as presented in Fig. 1, postulates three weighting systems related to (stimulus) selection, perceptual/semantic analysis, and response (selection) processes. However, the experimental work carried out thus far has dealt primarily with the first two (selection and analysis) weighting systems. This is mainly due to the need to demonstrate that criterion-specific effects originate from multiple (i.e., more than one) mechanisms. Follow-up work will address all three systems and their interplay.
}

in their search detection task (50 vs. $20 \mathrm{~ms}$ ), which is in accordance with the pattern observed in the present study. Furthermore, the differential DRE magnitude between the search and non-search detection tasks is compatible with the MWS hypothesis. If the DREs were indeed generated by different weighting systems $\left(\mathrm{S}_{\omega}\right.$ or $\left.\mathrm{A}_{\omega}\right)$ in the search and non-search detection tasks, differences between the tasks can be readily explained by assuming that $S_{\omega}$ and $A_{\omega}$ systems can give rise to DREs of different magnitudes; in fact, there is no reason to assume that all systems give rise to effects of the same magnitude.

Finally, large analysis-related DREs (relative to selectionrelated DREs) also exhibit greater variability (again, relative to selection-based effects). Given this, variability in analyses-based DREs might have "overshadowed" selection-based DREs in the search discrimination task, preventing the manifestation of a difference in DREs between search discrimination (both $\mathrm{S}_{\omega}$ and $\mathrm{A}_{\omega}$ mechanisms) and non-search detection (just $\mathrm{A}_{\omega}$ ) tasks in Experiment 2. Thus, markers of different dimension-weighting mechanisms might not only be the relative magnitude, but also the variability of the DREs they produce.

\section{Single-mechanism explanations}

Selection-based accounts Search-based accounts can account for only part of the present findings: Our search tasks (detection and discrimination) both used multi-item displays, which presumably influenced processes of stimulus selection. Thus, the significant DREs across task switches in Experiment 1 (search detection and search discrimination) could be explained by weighting of stimulus selection. However, even though stimulus selection processes were minimized for the non-search detection task, it did generate significant DREs. An exclusive selection-based explanation would encounter difficulty explaining the results for nonsearch task of Experiment 2 (non-search detection and search discrimination).

Response-based accounts Response-based accounts of DREs (Cohen \& Magen, 1999; Cohen \& Shoup, 1997, 2000; Feintuch \& Cohen, 2002) assume that these effects arise at the postselective processing stage of response selection. Evidence in support of this assumption stems from single-task paradigms in which the S-R mapping was fixed throughout the experimental trials. By contrast, in the present Experiments 1 and 2, switching between different tasks entailed switching between S-R mappings. To explain the significant DREs across task (and S-R-mapping) switches in Experiments 1 and 2, response-based accounts would have to assume a response selection process that works relatively independently of the particular S-R mapping; to our knowledge, no such claim has ever been made in 
the literature. Furthermore, a response-based mechanism thus modified would predict significant DREs across search and non-search detection tasks in which the S-R mapping is identical (target-present/absent response) - a prediction we repeatedly failed to substantiate (Rangelov et al., 2011b). Finally, it is not immediately clear why the same response-based DRE mechanism would produce weaker effects for the search detection task (Experiment 1) than for the non-search detection task (Experiment 2). To conclude, while some, as yet unspecified, modification of the response-based account might account for DREs across task switches, a single response-based mechanism of DREs would fail to account for all available findings.

Identification-based account The identification-based account proposed by Becker (2010) appears to be the only singlemechanism account compatible with the results of Experiments 1 and 2. From this perspective, target identification processes were involved in all tasks (search and non-search detection, and search discrimination), thus producing DREs across both task repetition and task switch trials. Furthermore, since perceptual analysis is not as demanding in the search detection task as in the other two tasks (determining the singleton status of the selected item suffices for the response!), the identification account could also explain the observed differences in the DRE magnitude between a task with a simple response criterion (search detection) and tasks with complex criteria (non-search detection and search discrimination). However, as has already been discussed in the Introduction, the identification account would also predict DREs across search detection and nonsearch discrimination tasks (examined in Experiments 1 and 2 of Rangelov et al., 2011a), as well as across search detection and non-search detection tasks (examined in Experiments 2A and 2B of Rangelov et al., 2011b); the results of our experiments repeatedly failed to substantiate these predictions. To conclude, despite being compatible with the present findings, the identification account fails to explain previously reported results. By contrast, the MWS hypothesis offers an explanatory framework compatible with both the present and the previous findings.

\section{Ambiguity resolution account revisited}

The ambiguity resolution account of Olivers and Meeter (2006, 2008; Meeter \& Olivers, 2006) assumes that behaviorally comparable DREs may have different sources-for instance, selection ambiguity or response ambiguity. In this respect, the ambiguity account is similar to the MWS hypothesis. The similarities, however, end at this point. The MWS hypothesis was devised to account for DREs observed across both task repetitions and task switches.
By contrast, the ambiguity resolution account was developed to explain which paradigms would be more likely to produce DREs than others. In order to advance our understanding of DREs across task switches, the latter account would have to take a clear stance with regard to how ambiguities that originate at different processing stages are combined to influence sequential task performance; yet, to our knowledge, no such a clarification has been provided to date.

To illustrate, from its present state of theoretical elaboration, it is not immediately clear whether or not the ambiguity resolution account would predict significant DREs across two tasks, one of which is ambiguous with regard to stimulus selection (e.g., search detection) and the other with regard to response selection (e.g., non-search detection). One possibility is that ambiguity is pooled across all its sources (i.e., selection, response, task set), in which case sufficiently strong ambiguity in one task, whatever its source, should result in DREs across switches to another task of sufficiently strong ambiguity, again regardless of the source of ambiguity in the second task. Consequently, source-independent pooling of ambiguities would predict DREs across any two tasks of sufficient ambiguity-a prediction that our work repeatedly failed to support (Rangelov et al., 2011a, 2011b).

The other alternative would be to assume sourcedependent propagation of ambiguities; that is, ambiguity at one processing stage (e.g., stimulus selection) will propagate and produce DREs on the next trial, independently of ambiguity at a different processing stage (e.g., response selection). On this assumption, DREs would be predicted to manifest across tasks that share a source of ambiguity, but not across tasks with different sources of ambiguity. This alternative would be compatible with most of the reported findings. However, the assumption of sourcedependent ambiguity propagation is just a restatement, in terms of ambiguity, of the already developed MWS hypothesis.

To conclude, further theoretical development would be necessary for the ambiguity account to be able to explain the set of reported findings. In its present state, it is equally compatible with both falsified and confirmed DREs across task switches.

Relation to the task-switching literature

In the Introduction, different types of intertrial effect were conceptualized as being either feature or criterion specific in nature, with DREs being of the latter type. In the following section, we argue that, like DREs, TSCs are, at least to some extent, criterion specific, too, and go on to elaborate the implications of the MWS hypothesis, devised to account for DREs, for theories of task switching. 
The core property of task switch paradigms is that observers are asked to perform two (or more) different tasks, with the specific task to be executed varying across trials. Most frequently, the two tasks use the same set of stimuli, while the decisions (and responses) are made to vary across tasks. Thus, across two trials, the task can either repeat or switch (with observers being informed of the specific task to be performed on a given trial by a precue). Similar to DREs, switching tasks across trials elevates RTs and decreases accuracy, relative to task repetitions. Most important, task switches always involve switches of criteria for either visual selection of the taskrelevant item amongst nonrelevant items (Mayr \& Keele, 2000) or of criteria for the perceptual/semantic analysis of an item presented in isolation (Logan \& Schneider, 2006; Meiran, 1996), or both (Rogers \& Monsell, 1995). On the other hand, repeating the exact stimulus and/or the required response across task switches has typically been found to have either little effect on or to be detrimental to performance (e.g., Meiran, 1996; Rogers \& Monsell, 1995), arguing that task-switching effects are criterion rather than feature specific in type. In this respect (i.e., criterion specificity), TSCs are comparable to the DREs observed in visual search tasks. A notable difference, however, between task-switching and dimension repetition effects is that task switches usually also entail a switch in S-R mappings (but see Mayr \& Keele, 2000), while DREs are typically investigated using an $\mathrm{S}-\mathrm{R}$ mapping that is fixed throughout the trials of a task

To the extent to which TSCs can be conceived of as criterion-specific effects, the MWS hypothesis is also relevant for the task-switching literature. First, the present experiments provide evidence that the time necessary to switch between different tasks (e.g., search detection and search discrimination) can be shortened, provided the task-relevant dimension repeats across different tasks (i.e., significant DREs across task switches). Second, previously reported experiments (Rangelov et al., 2011a, 2011b) demonstrate that for DREs to arise across task switches, the tasks in question must share at least one of the postulated weighting mechanisms. Importantly, according to the MWS hypothesis, the very same weighting mechanisms operate both within and across tasks. An implication of the MWS hypothesis for the taskswitching literature is that switching between tasks resembles processing within a task: DREs as an indicator of criterion-specific control mechanisms persist both within and across tasks. This implies that task sets, defined as "the configuration of perceptual, attentional, mnemonic, and motor processes critical for a particular task goal" (Mayr \& Keele, 2000, p. 5), are a composite entity consisting of relatively independent configuration settings for different processes, and, critically, that these configuration settings carry over across different tasks that use similar processes. Finally, the MWS hypothesis is also in accordance with recent ideas in the task-switching literaturenamely, that the processes regulating behavior within a task are closely related to those that govern switching between tasks (Logan \& Schneider, 2006; Logan, Schneider, \& Bundesen, 2007; Schneider \& Logan, 2009).

In summary, we contend that, analogous to DREs, TSCs too arise as a consequence of the requirement to switch, across two trials, a criterion for selection, analysis, or responding. The most important implications of the MWS hypothesis for understanding task switching are that (1) criteria for selection, analysis, and responding can be configured independently of each other by virtue of independent mechanisms controlling these processes, and (2) the same sequence-sensitive mechanisms $\left(\mathrm{S}_{\omega}, \mathrm{A}_{\omega}, \mathrm{R}_{\omega}\right)$ can control performance in both task-switching and fixed-task paradigms. Accordingly, the MWS hypothesis, in parallel to some recent accounts of task-switching processes (e.g., Logan et al., 2007), offers a unified perspective on within- and across-task processes of cognitive control, in contrast to a more traditional approach that hypothesizes distinct executive processes specifically engaged in task-switching and absent in fixed-task paradigms (e.g., Mayr \& Keele, 2000; Meiran, 1996; Rogers \& Monsell, 1995).

\section{Conclusions}

Review of findings on criterion-specific intertrial effects in different psychophysical paradigms suggested that the single-mechanism accounts proposed in the literature cannot fully explain all the available data. To fill this explanatory gap, a multiple-weighting-systems (MWS) hypothesis was developed. On this hypothesis, there exist several independent mechanisms that can all produce DREs, by virtue of being sensitive to (sequences in) perceptual dimensions. Previous findings, based on a task switch approach, demonstrated a behavioral dissociation between sources of DREs in search and non-search paradigms. In the present study, using a similar approach, we demonstrated that a single task can engage multiple weighting mechanisms at the same time. Although separable, these weighting systems are all situated along the same processing path, leading from stimulus selection through perceptual analysis to response selection and response execution.

Acknowledgments The research was supported by LMU excellent (research professorship funding) to H.J.M, and by the following research grants: German cluster of excellence Cognition for Technical Systems (CoTeSys, EC 142) and German Israeli Foundation (GIF) to H.J.M and M.Z. 
The authors would also like to thank Stefanie Becker and Karen Mortier for their useful comments on the earlier versions of the manuscript.

\section{References}

Becker, S. I. (2008). Can intertrial effects of features and dimensions be explained by a single theory? Journal of Experimental Psychology: Human Perception and Performance, 34, 14171440. doi:10.1037/a0011386

Becker, S. I. (2010). Testing a postselectional account of acrossdimension switch costs. Psychonomic Bulletin \& Review, 17, 853-861. doi:10.3758/PBR.17.6.853

Cohen, A., \& Magen, H. (1999). Intra- and cross-dimensional visual search for single-feature targets. Perception \& Psychophysics, 61, 291-307.

Cohen, A., \& Shoup, R. (1997). Perceptual dimensional constraints in response selection processes. Cognitive Psychology, 32, 128-181. doi:10.1006/cogp. 1997.0648

Cohen, A., \& Shoup, R. (2000). Response selection processes for conjunctive targets. Journal of Experimental Psychology: Human Perception and Performance, 26, 391-411.

Druey, M. D., \& Hübner, R. (2008). Response inhibition under task switching: Its strength depends on the amount of task-irrelevant response activation. Psychological Research, 72, 515-527. doi:10.1007/s00426-007-0127-1

Fecteau, J. H. (2007). Priming of pop-out depends upon the current goals of observers. Journal of Vision, 7(6, Art. 1), 1, 1-11. doi: $10.1167 / 7.6 .1$

Feintuch, U., \& Cohen, A. (2002). Visual attention and coactivation of response decisions for features from different dimensions. Psychological Science, 13, 361-369.

Found, A., \& Müller, H. J. (1996). Searching for unknown feature targets on more than one dimension: Investigating a "dimensionweighting" account. Perception \& Psychophysics, 58, 88-101.

Gade, M., \& Koch, I. (2007). The influence of overlapping response sets on task inhibition. Memory \& Cognition, 35, 603-609. doi:VL-35

Gramann, K., Töllner, T., \& Müller, H. J. (2010). Dimension-based attention modulates early visual processing. Psychophysiology, 47, 968-978. doi:10.1111/j.1469-8986.2010.00998.x

Koch, I., Gade, M., Schuch, S., \& Philipp, A. M. (2010). The role of inhibition in task switching: A review. Psychonomic Bulletin \& Review, 17, 1-14. doi:10.3758/PBR.17.1.1

Krummenacher, J., Grubert, A., \& Müller, H. J. (2010). Inter-trial and redundant-signals effects in visual search and discrimination tasks: Separable pre-attentive and post-selective effects. Vision Research, 50, 1382-1395. doi:10.1016/j.visres.2010.04.006

Logan, G. D., \& Schneider, D. W. (2006). Priming or executive control? Associative priming of cue encoding increases "switch costs" in the explicit task-cuing procedure. Memory \& Cognition, 34, 1250-1259.

Logan, G. D., Schneider, D. W., \& Bundesen, C. (2007). Still clever after all these years: Searching for the homunculus in explicitly cued task switching. Journal of Experimental Psychology: Human Perception and Performance, 33, 978-994. doi:10.1037/0096-1523.33.4.978
Mayr, U. (2001). Age differences in the selection of mental sets: The role of inhibition, stimulus ambiguity, and response-set overlap. Psychology and Aging, 16, 96-109. doi:10.1037/08827974.16.1.96

Mayr, U., \& Keele, S. W. (2000). Changing internal constraints on action: The role of backward inhibition. Journal of Experimental Psychology: General, 129, 4-26.

Meeter, M., \& Olivers, C. N. L. (2006). Intertrial priming stemming from ambiguity: A new account of priming in visual search. Visual Cognition, 13, 202-222. doi:10.1080/13506280500277488

Meiran, N. (1996). Reconfiguration of processing mode prior to task performance. Journal of Experimental Psychology: Learning, Memory, and Cognition, 22, 1423-1442.

Mortier, K., Theeuwes, J., \& Starreveld, P. (2005). Response selection modulates visual search within and across dimensions. Journal of Experimental Psychology: Human Perception and Performance, 31, 542-557. doi:10.1037/0096-1523.31.3.542

Müller, H. J., Heller, D., \& Ziegler, J. (1995). Visual search for singleton feature targets within and across feature dimensions. Perception \& Psychophysics, 57, 1-17.

Müller, H. J., \& Krummenacher, J. (2006a). Locus of dimension weighting: Preattentive or postselective? Visual Cognition, 14, 490-513.

Müller, H. J., \& Krummenacher, J. (2006b). Visual search and selective attention. Visual Cognition, 14, 389-410.

Olivers, C. N. L., \& Humphreys, G. W. (2003). Attentional guidance by salient feature singletons depends on intertrial contingencies. Journal of Experimental Psychology: Human Perception and Performance, 29, 650-657.

Olivers, C. N. L., \& Meeter, M. (2006). On the dissociation between compound and present/absent tasks in visual search: Intertrial priming is ambiguity driven. Visual Cognition, 13, 1-28.

Olivers, C. N. L., \& Meeter, M. (2008). Feature priming in visual search does not depend on the dimensional context. Visual Cognition, 16, 785-803. doi:10.1080/13506280701428658

Rangelov, D., Müller, H. J., \& Zehetleitner, M. (2011a). Dimensionspecific intertrial priming effects are task-specific: Evidence for multiple weighting systems. Journal of Experimental Psychology: Human Perception and Performance, 37, 100-114. doi:10.1037/ a0020364

Rangelov, D., Müller, H. J., \& Zehetleitner, M. (2011b). Independent dimension-weighting mechanisms for visual selection and stimulus identification. Journal of Experimental Psychology: Human Perception and Performance, 37, 1369-1382. doi:10.1037/a0024265

Rogers, R. D., \& Monsell, S. (1995). Costs of a predictable switch between simple cognitive tasks. Journal of Experimental Psychology: General, 124, 207-231.

Schneider, D. W., \& Logan, G. D. (2009). Selecting a response in task switching: Testing a model of compound cue retrieval. Journal of Experimental Psychology: Learning, Memory, and Cognition, 35, 122-136. doi:10.1037/a0013744

Töllner, T., Gramann, K., Muller, H. J., Kiss, M., \& Eimer, M. (2008). Electrophysiological markers of visual dimension changes and response changes. Journal of Experimental Psychology, 34, 531-542. doi:10.1037/0096-1523.34.3.531 